\title{
Clinicopathologic and Oncological Outcomes in Korean Men With Advanced Metastatic Testicular Cancer Undergoing Postchemotherapeutic Retroperitoneal Lymph Node Dissection
}

\author{
Hyeong Dong Yuk ${ }^{1}$, Minyong Kang ${ }^{1,2}$, Jung Keun Lee ${ }^{3}$, Sung Kyu Hong ${ }^{3}$, \\ Ja Hyeon Ku', Seok-Soo Byun ${ }^{3}$, Cheol Kwak', Hyeon Hoe Kim', \\ Sang Eun Lee ${ }^{3}$, Chang Wook Jeong ${ }^{1}$ \\ ${ }^{1}$ Department of Urology, Seoul National University Hospital, Seoul, Korea \\ ${ }^{2}$ Department of Urology, Samsung Medical Center, Seoul, Korea \\ ${ }^{3}$ Department of Urology, Seoul National University Bundang Hospital, Seongnam, Korea
}

\begin{abstract}
Purpose: To evaluate the clinicopathologic and oncological outcomes of advanced metastatic testicular cancer in Korean men who underwent retroperitoneal lymph node dissection (RPLND) following chemotherapy.

Materials and Methods: Data of 26 patients with testicular cancer who underwent RPLND after chemotherapy at 2 hospitals in Korea between September 2004 and June 2016 were retrospectively analyzed. Clinical and histopathological variables such as stage of the testicular cancer, age of the patients during surgery, size of the retroperitoneal lymph nodes (RPLNs), histopathological results, duration and complications related to the surgery, cancer recurrence, and mortality were analyzed.

Results: During testicular surgery, the T stage was pT1, pT2, and pT3 in 50\% ( $n=13), 26.9 \%(n=7)$, and $15.3 \%$ $(n=4)$ of the patients, respectively. Mixed germ cell tumor was the most common finding, seen in $73.1 \%(n=19)$ of patients. The indications for RPLND were residual lymph nodes after chemotherapy, $84.6 \%(n=22)$; and disease progression and remission, $7.7 \%(n=2)$. Pathological analysis revealed viable tumors in $19.2 \%$ of patients $(n=5)$, necrotic/fibrotic tissue in $42.3 \%(n=11)$, and teratoma in $34.6 \%(n=9)$. Intraoperative and postoperative complications occurred in $23.1 \%(n=6)$ and $19.2 \%$ of patients $(n=5)$. The median duration of follow-up was 27.5 months (interquartile range, $1.3-108.2$ months); $11.5 \%(n=3)$ patients had recurrence, and $3.8 \%(n=1)$ died of progressive metastatic testicular cancer.

Conclusions: Viable germ cell tumors were present in $19.2 \%$ of patients with testicular cancer who underwent RPLND after chemotherapy. This is the first study of its kind in the Korean population. (Korean J Urol Oncol 2017;15:143-151)
\end{abstract}

Key Words: Testicular germ cell tumor $\cdot$ Postoperative complications $\cdot$ Lymph node excision

Received October 17, 2017, Revised November 23, 2017,

Accepted December 6, 2017

Corresponding Author: Chang Wook Jeong

Department of Urology, Seoul National University Hospital, 101

Daehak-ro, Jongno-gu, Seoul 03080, Korea

E-mail: drboss@ korea.com

Tel: +82-2-2072-3899, Fax: +82-2-742-4665

ORCID code: https://orcid.org/0000-0002-2200-5019

\section{INTRODUCTION}

Testicular cancer is a rare cancer occurring in men, with an incidence of approximately $1 \%{ }^{1}$ However, it is one of the most common cancers among young men, and in Western countries, it is the most common urogenital cancer occurring in men between the ages of 20 and 40 years. The disease is also the sec-
\end{abstract}

This is an Open Access article distributed under the terms of the Creative Commons Attribution Non-Commercial License (http://creativecommons.org/licenses/by-nc/4.0/) which permits unrestricted non-commercial use, distribution, and reproduction in any medium, provided the original work is properly cited. 2017 (C) Copyright The Korean Urological Oncology Society and The Korean Prostate Society. All Rights Reserved. 
ond most widespread cancer among male adolescents, after leukemia. ${ }^{2}$ Although the mortality rate is low, the incidence of testicular cancer is reportedly increasing in Western populations. ${ }^{3}$ According to the National Cancer Information Center in Korea, 230 cases of testicular cancer were reported in 2014. The incidence in Korea is reportedly less than that in Western countries; however, it is increasing in a manner similar to that in Western countries. ${ }^{4}$ Germ cell tumors (GCTs) are highly curable cancers when appropriately treated with multimodal therapy. Depending on the stage of the disease, orchiectomy, chemotherapy, and retroperitoneal lymph node dissection (RPLND) are used in combination. ${ }^{5}$ Lymph node metastasis from testicular cancer occurs primarily to the lymph nodes around the large vessels of the retroperitoneal cavity. The commonly involved lymph nodes are the precaval, paracaval, para-aortic, and preaortic lymph nodes. ${ }^{6}$ RPLND is commonly carried out after surgery and first-line chemotherapy in patients with metastatic testicular cancer, especially in those with advanced stage $\mathrm{T} 2 \mathrm{~b}$ disease. The procedure is indicated in patients who do not achieve complete remission after primary chemotherapy or cases in which the tumor contains a teratoma component. In patients with a metastatic GCT, residual retroperitoneal mass lesions persist in $2 \%-50 \%$ of the patients following chemotherapy. ${ }^{7-9}$ In such cases, RPLND is used as the primary treatment. ${ }^{10}$ RPLND can be performed through various approaches, which include open, laparoscopic, and robot-assisted procedures. ${ }^{11}$ The overall long-term relapse-free survival after complete RPLND is reported to be over $90 \% .^{12}$ If residual tissue remains, the risk of relapse increases, and the disease-specific survival rate is only $21 \% .{ }^{13}$ However, there have been no such reports of patients in Korea.

The purpose of this study was to evaluate the clinicopathologic and oncological outcomes of advanced metastatic testicular cancer in Korean men who underwent RPLND following chemotherapy. Additionally, we sought to evaluate the treatment and prognosis of patients with metastatic testicular cancer in Korea.

\section{MATERIALS AND METHODS}

We retrospectively collected and analyzed the data of patients who underwent RPLND after chemotherapy at the Seoul National University Hospital and the Seoul National University Bundang Hospital between September 2004 and June 2016.
Data of 26 patients were included in this analysis. The most common systemic chemotherapy regimen administered was bleomycin, etoposide, and cisplatin (BEP) and was administered over 3 to 4 cycles. The indications for RPLND after chemotherapy in these patients were residual lymph nodes after chemotherapy, disease progression, and salvage after chemotherapy. Most patients underwent open RPLND, but several underwent robot- and hand-assisted laparoscopic RPLND. Follow-up physical examination, chest radiography, abdominal and pelvic computed tomography (CT), and measurement of serum tumor markers (human chorionic gonadotropin, $\alpha$-fetoprotein, and lactate dehydrogenase) were performed every 3 months during the first 2 years and every 6 months during the third year. Subsequently, patients were followed up annually for up to 5 years postoperatively.

We analyzed clinical and pathological variables such as stage of the testicular cancer, age of the patients during surgery, size of the retroperitoneal lymph nodes (RPLNs), histopathological results of the testicular resection and RPLND, method followed for the RPLND, time taken for surgery, and operative complications. In addition, the oncological outcomes, such as the recurrence rate after RPLND, and the incidence of cancer-specific death were analyzed. Predictive factors were analyzed using multivariate logistical regression. The primary endpoint was the pathological result after RPLND, and the secondary endpoints were the oncological outcomes.

\section{RESULTS}

All 26 patients underwent RPLND after first-line chemotherapy. Patient characteristics are shown in Table 1. The median size of the largest RPLN was $2.75 \mathrm{~cm}$ (range, 2.20-4.50 cm), and the interquartile range (IQR) was $2.42-7.00 \mathrm{~cm}$ before and after chemotherapy. Twenty patients (76.9\%) had an RPLN $\geq 3 \mathrm{~cm}$ and 11 patients $(42.3 \%)$ had metastasis to other sites as well. Most patients underwent platinum-based chemotherapy. BEP was the most commonly used chemotherapy regimen (18 patients, 69.2\%). In addition, paclitaxel, ifosfamide, and cisplatin, and etoposide, ifosfamide, and cisplatin (VIP) regimens were also used. Twenty-one patients $(80.7 \%)$ underwent 3 to 4 cycles of BEP chemotherapy. Three patients (11.5\%) underwent 4 cycles of VIP chemotherapy. The remaining patients received alternative individualized chemotherapy, mostly combinations of BEP and VIP (Table 2). 
Table 1. Characteristics of patients with testicular cancer undergoing postchemotherapy retroperitoneal lymph node dissection (RPLND) $(\mathrm{n}=26)$

\begin{tabular}{|c|c|}
\hline Variable & Value \\
\hline Age (yr) at RPLND & $25.5(22.2-33.2)$ \\
\hline Body mass index $\left(\mathrm{kg} / \mathrm{m}^{2}\right)$ & $23.03(19.85-25.74)$ \\
\hline \multicolumn{2}{|l|}{ ECOG performance status } \\
\hline 0 & $21(80.8)$ \\
\hline 1 & $5(19.2)$ \\
\hline \multicolumn{2}{|l|}{ Primary tumor laterality } \\
\hline Right & $13(50.0)$ \\
\hline Left & $13(50.0)$ \\
\hline Primary tumor size $(\mathrm{cm})$ & $4.25(2.82-6.65)$ \\
\hline \multicolumn{2}{|l|}{ Pathologic $\mathrm{T}$ stage } \\
\hline pT0 & $1(4.0)$ \\
\hline pT1 & $13(52.0)$ \\
\hline pT2 & $7(28.0)$ \\
\hline pT3 & $4(16.0)$ \\
\hline Missing & $1 / 26$ \\
\hline \multicolumn{2}{|l|}{ Primary tumor stage (AJCC) } \\
\hline I & $3(11.5)$ \\
\hline II & $5(19.2)$ \\
\hline III & $18(69.2)$ \\
\hline \multicolumn{2}{|l|}{ Primary tumor pathology } \\
\hline Seminoma & $2(7.7)$ \\
\hline Nonseminoma & $5(19.2)$ \\
\hline Mixed-GCT & $19(73.1)$ \\
\hline \multicolumn{2}{|l|}{ Regimen of chemotherapy } \\
\hline $\mathrm{BEP}$ & $18(69.2)$ \\
\hline VIP & $3(19.2)$ \\
\hline Combined & $3(73.1)$ \\
\hline \multicolumn{2}{|l|}{ Maximal nodal size $(\mathrm{cm})$} \\
\hline Prechemotherapy & $4.45(2.42-7.00)$ \\
\hline Postchemotheraphy (before RPLND) & $2.75(2.20-4.50)$ \\
\hline \multicolumn{2}{|l|}{ Indication of RPLND } \\
\hline Residual mass after chemotherapy & $22(84.6)$ \\
\hline Progression after chemotherapy & $2(7.7)$ \\
\hline Salvage treatment & $2(7.7)$ \\
\hline \multicolumn{2}{|l|}{ Operation type } \\
\hline Open & $21(80.8)$ \\
\hline HALS & $2(7.7)$ \\
\hline Robot-assisted & $3(11.5)$ \\
\hline Operation time (min) & $192.5(152.5-267.5)$ \\
\hline Estimated blood loss (mL) & $275.0(100-387.5)$ \\
\hline Adjuvant procedures during surgery & $9(34.6)$ \\
\hline \multicolumn{2}{|l|}{ RPLND pathology } \\
\hline Viable GCT & $5(19.2)$ \\
\hline Fibrotic tissue & $11(42.3)$ \\
\hline Mature teratoma & $10(38.5)$ \\
\hline Hospital stay (day) & $9(7-10)$ \\
\hline \multicolumn{2}{|l|}{ Complications } \\
\hline Intraoperative & $6(23.1)$ \\
\hline Postoperative & $5(19.2)$ \\
\hline Recurrence & $3(11.5)$ \\
\hline
\end{tabular}

Table 1. Continued

\begin{tabular}{ll}
\hline \multicolumn{1}{c}{ Variable } & \multicolumn{2}{c}{ Value } \\
\hline Mortality & \\
All-cause & $1(3.8)$ \\
Cancer-specific & $1(3.8)$ \\
Follow-up duration (mo) & $27.5(1.3-108.2)$ \\
\hline
\end{tabular}

Values are presented as median (range) or number $(\%)$. ECOG: eastern cooperative oncology group, AJCC: American Joint Committee on Cancer, GCT: germ cell tumor, BEP: bleomycin/etoposide/cisplatin, VIP: etoposide/ifosfamide/cisplatin, HALS: hand assisted laparoscopic surgery.

Twenty-one patients (80.8\%) had an Eastern Cooperative Oncology Group (ECOG) performance status of grade 0, and $5(19.2 \%)$ had an ECOG performance status of grade 1. The location of the primary tumor was the left testicle in 13 patients $(50 \%)$ and the right testicle in 13 patients $(50 \%)$. The median size of the primary tumor was $4.25 \mathrm{~cm}$ (range, $2.82-6.65 \mathrm{~cm}$ ). At the time of testicular surgery, the most common $\mathrm{T}$ stage was $\mathrm{T} 1$, with stages $\mathrm{pT} 1, \mathrm{pT} 2$, and $\mathrm{pT} 3$ present in 13 patients (50\%), 7 patients (26.9\%), and 4 patients $(15.3 \%)$, respectively. The histopathology of the primary tumor demonstrated a mixed GCT in 19 patients (73.1\%), a nonseminomatous GCT in 5 patients $(19.2 \%)$, and a seminoma in 2 patients $(7.7 \%)$. As per the American Joint Committee on Cancer staging system, the disease stage at presentation was stage I in 3 patients (11.5\%), stage II in 5 patients (19.2\%), and stage III in 18 patients (69.2\%). On CT, enlarged lymph nodes were found in the preaortic $(76.9 \%)$, para-aortic $(53.8 \%)$, interaortocaval (11.5\%), paracaval $(11.5 \%)$, and pelvic $(23 \%)$ sites. In addition, one patient had renal hilar lymph node enlargement and 1 patient had retrocrural lymph node enlargement. The most common distant metastatic site was the lungs (12 patients, $46.1 \%$ ). Liver metastasis was present in 1 patient $(3.8 \%)$. The mean postoperative hospital stay was 9 days (range, 6-12 days).

The indications for RPLND were as follows: residual lymph nodes after chemotherapy in 22 patients (84.6\%); disease progression and remission in 2 patients (7.7\%); and salvage in 2 patients $(7.7 \%)$. A total of 21 patients $(80.8 \%)$ underwent open RPLND, 3 (11.5\%) underwent robot-assisted RPLND, and 2 (7.7\%) underwent hand-assisted laparoscopic RPLND. The mean time taken for surgery was 190 minutes (range, 120-400 minutes). Vascular injury was the most common intraoperative complication; inferior mesenteric artery sacrifice, inferior mesenteric vein sacrifice, inferior vena cava injury, and renal artery 


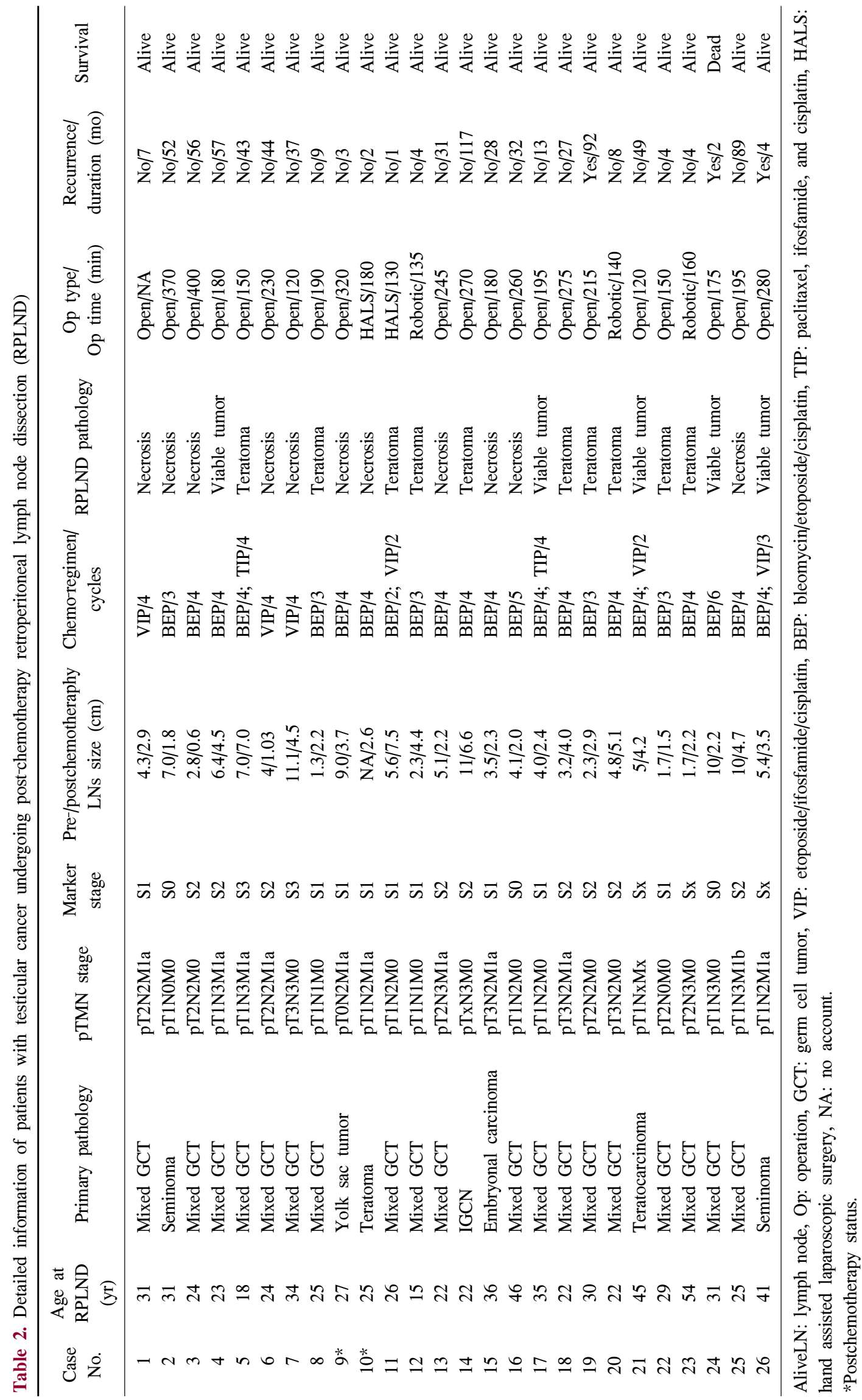


injury each occurred in 1 patient (15.3\%). One patient also had a splenic injury (3.8\%). Adjuvant surgery was performed in 7 patients (26.9\%); 3 patients (11.5\%) underwent vascular surgery, and 1 patient $(3.8 \%)$ each underwent nephrectomy, splenectomy, and video-assisted thoracoscopic metastasectomy. Postoperative complications included pleural effusion in 1 patient (3.8\%), pneumothorax in 2 patients $(7.7 \%)$, chylous ascites in 1 patient $(3.8 \%)$, and arm weakness in 1 patient $(3.8 \%)$. Histopathological analysis of the lymph nodes dissected during RPLND revealed viable tumors in 5 patients (19.2\%). Necrotic/fibrotic tissue and teratoma were found in 11 patients (42.3\%) and 10 patients (38.5\%), respectively. Intraoperative and postoperative complications occurred in 6 patients (23.1\%) and 5 patients (19.2\%), respectively (Table 2).

The median follow-up duration was 27.5 months (IQR, 1.3108.2 months). Three patients experienced a recurrence
(11.5\%), and 1 patient $(3.8 \%)$ died of progressive metastatic testicular cancer. Two of the 3 patients who had recurrence were found to have viable tumors at RPLND, and 1 of these patients died (the only death in our study). Patients with recurrence presented with interaortocaval lymph node, preaortic lymph node, para-aortic lymph node, and common iliac lymph node metastasis. At the time of recurrence, 2 patients had elevated lactate dehydrogenase (LDH) levels, and elevated LDH and alpha fetoprotein levels, respectively, and one had no elevated biomarker levels. The pathological results after RPLND for these patients were necrosis, viable tumor, and teratoma. One of 3 patients with recurrence underwent additional radiation therapy, and 2 underwent further observation.

Table 3 shows the response to preoperative chemotherapy according to pathology results of RPLND specimens. In the viable tumor group, the mean lymph node size before chemo-

Table 3. Outcomes after chemotherapy according to pathology results after retroperitoneal lymph node dissection (RPLND)

\begin{tabular}{|c|c|c|c|c|}
\hline RPLND pathology & Necrosis $(n=11)$ & Viable tumor $(\mathrm{n}=5)$ & Teratoma $(n=10)$ & $\mathrm{p}^{\text {-value }}$ \\
\hline \multicolumn{5}{|l|}{ Lymph node size } \\
\hline Prechemotherapy & $5.6 \pm 2.6$ & $7.2 \pm 2.8$ & $3.9 \pm 3.0$ & 0.217 \\
\hline Postchemotherapy & $2.6 \pm 1.3$ & $4.1 \pm 2.0$ & $4.0 \pm 2.0$ & 0.062 \\
\hline Lymph node change & & & & 0.001 \\
\hline No change & $0(0)$ & $1(20.0)$ & $0(0)$ & \\
\hline Increase & $0(0)$ & $0(0)$ & $7(70.0)$ & \\
\hline Decrease & $11(100)$ & $4(80.0)$ & $3(30.0)$ & \\
\hline \multicolumn{5}{|l|}{ Lymph node location } \\
\hline \multicolumn{5}{|l|}{ Prechemotherapy } \\
\hline Preaortic & $0(0)$ & $1(20.0)$ & $1(10.0)$ & 0.357 \\
\hline Para-aortic & $10(90.9)$ & $4(80.0)$ & $7(70.0)$ & 0.478 \\
\hline Interaortocaval & $7(63.6)$ & $5(100.0)$ & $3(30.0)$ & 0.031 \\
\hline Para-caval & $1(9.1)$ & $2(40.0)$ & $1(10.0)$ & 0.236 \\
\hline Renal hilar & $1(9.1)$ & $0(0)$ & $0(0)$ & 0.492 \\
\hline Pelvic & $3(27.3)$ & $0(0)$ & $2(20.0)$ & 0.438 \\
\hline \multicolumn{5}{|l|}{ Postchemotherapy } \\
\hline Preaortic & $0(0)$ & $1(20.0)$ & $0(0)$ & 0.113 \\
\hline Para-aortic & $9(81.8)$ & $4(80.0)$ & $7(70.0)$ & 0.800 \\
\hline Interaortocaval & $8(72.7)$ & $3(60.0)$ & $2(20.0)$ & 0.048 \\
\hline Para-caval & $1(9.1)$ & $1(20.0)$ & $0(0)$ & 0.381 \\
\hline Renal hilar & $1(9.1)$ & $0(0)$ & $0(0)$ & 0.492 \\
\hline Pelvic & $3(27.3)$ & $0(0)$ & $2(20.0)$ & 0.438 \\
\hline AFP prechemotherapy & $1,356.6 \pm 2,287.9$ & $361.6 \pm 333.8$ & $1,433.2 \pm 2,589.8$ & 0.953 \\
\hline AFP postchemotherapy & $7.0 \pm 7.2$ & $175.8 \pm 232.9$ & $2.8 \pm 1.6$ & 0.984 \\
\hline hCG prechemotherapy & $22,658.9 \pm 60,981.8$ & $21,941.5 \pm 45,919.4$ & $2,376.4 \pm 7,107.7$ & 0.306 \\
\hline hCG postchemotherapy & $2.0 \pm 1.9$ & $2.6 \pm 0.9$ & $2.3 \pm 0.9$ & 0.636 \\
\hline LDH prechemotherapy & $638.6 \pm 854.3$ & $571.6 \pm 421.8$ & $313.4 \pm 197.4$ & 0.223 \\
\hline LDH postchemotherapy & $152.6 \pm 81.7$ & $223.8 \pm 75.3$ & $199.6 \pm 77.8$ & 0.187 \\
\hline
\end{tabular}

Values are presented as mean \pm standard deviation or number $(\%)$.

AFP: alpha fetoprotein, hCG: human chorionic gonadotropin, LDH: lactate dehydrogenase. 
therapy was relatively large. The lymph node size decreased after chemotherapy in the necrosis group and the viable tumor group, but increased in the teratoma group. Seven patients showed increased lymph node size. Recurrent lymph node metastases were relatively common in the interaortocaval and para-aortic areas. Biomarker levels decreased in all groups after chemotherapy. There were some patients who were not normalized in the viable tumor group. Post-RPLND specimens in most patients showed viable and teratoma tumors. In 1 patient with a viable tumor and teratoma, 2 cycles of the VIP regimen were performed as post-RPLND chemotherapy.

Multivariate logistic regression analysis was performed to determine predictive factors for viable tumors, but no significant factor was identified.

\section{DISCUSSION}

RPLND after chemotherapy was first reported by Comisarow and Grabstald ${ }^{14}$ about 40 years ago. The authors recommended RPLND for relapse of primary disease after RPLND or after chemotherapy. With improvements in the efficacy and delivery of chemotherapy, a more recent study found that the rate of residual GCT at the time of RPLND has decreased, and the ratio of fibrosis and necrosis has increased; however, there is no difference in the frequency of teratomas. ${ }^{15}$ Additionally, several studies have reported a difference in survival rates depending on the final pathological results. Several researchers have tried to determine the predictive factors for pathological outcomes. ${ }^{16}$ They reported that although size is not a predictor, residual masses of about $2-\mathrm{cm}$ size have potential for recurrence in $20 \%-33 \%$ of cases. This is the reason for performing RPLND when a residual mass is present. ${ }^{17}$

RPLND is associated with a low mortality rate, despite the complicated and difficult nature of the procedure. Gels et al. ${ }^{18}$ reported a mortality rate of $<1 \%$, while Nowroozi et al. ${ }^{19}$ reported a mortality rate of $0.8 \%-1 \%$. Five other larger studies reported mortality rates of $1 \%$. In agreement with the results in these reports, the mortality rate in our study was $1 \%$.

With respect to complications associated with RPLND after chemotherapy, Baniel et $\mathrm{al}^{20}$ and Considine et al. ${ }^{21}$ reported complication rates of $18 \%$ and $29.5 \%$, respectively. Maldonado-Valadez et al. ${ }^{22}$ and Nowroozi et al. ${ }^{19}$ reported complication rates of $57.1 \%$ and $20 \%-35 \%$, respectively. Additionally, Djaladat et al. ${ }^{23}$ and Steiner et al. ${ }^{24}$ reported com- plication rates of $35 \%$ and $43.8 \%$, respectively.

In our study, intraoperative (23.1\%) and postoperative complication rates $(11.5 \%)$ were not significantly different from those obtained in previous studies. Clavien-Dindo classification grades 3 and 4 complications occurred in $19.2 \%$ of our patients. However, a high incidence of intraoperative and postoperative complications reflects the complexity of RPLND surgery. RPLND after chemotherapy is more challenging due to the extensive connective tissue response and distortion of tissue planes caused by the tumor. After chemotherapy, the residual tumor can invade other structures including the ureters and major blood vessels, such as the aorta and the vena cava. Therefore, additional surgeries following RPLND, such as nephrectomy, aortic replacement, vena cava resection, and ureter repair, may be necessary. ${ }^{21}$ Djalada et al. $^{23}$ reported that $30 \%$ of patients underwent additional surgery; $15 \%$ of patients underwent vascular surgery, and $14 \%$ underwent nephrectomy. Considine et al. ${ }^{21}$ reported that $28 \%$ of patients required further surgery including vascular surgery in $2.6 \%$ and nephrectomy in $20 \%$ of patients. In our study, adjuvant surgery was performed in 7 patients $(26.9 \%) ; 11.5 \%$ underwent vascular surgery, and $3.8 \%$ underwent nephrectomy. Additionally, 1 patient each underwent lung surgery and splenic surgery.

With regards to pathology, several other studies have reported different prevalence rates for teratoma, necrosis and fibrosis, and viable tumors. Nakamura et al. ${ }^{25}$ and Alanee et al. ${ }^{26}$ reported a probability of $9.0 \%$ and $43 \%$ for the presence of viable tumors, respectively, while several other studies have also reported viable tumors in $10 \%-40 \%$ of patients. ${ }^{21-27}$ In our study, the frequency of viable tumors was $19.2 \%$, similar to that observed in previous studies. Compared to earlier studies, recent studies have shown that the percentage of patients with residual cancer after chemotherapy has declined, which could potentially be related to the improved efficacy and delivery of chemotherapy.

Fléchon et $\mathrm{al}^{27}$ reported a $4 \%$ relapse rate after postchemotherapy RPLND 26 and Considine et al. ${ }^{21}$ reported a relapse rate of $18.5 \%$ with a median follow-up of 6.1 months (range, 1.3-39 months). The relapse rate after postchemotherapy RPLND in our study was $11.5 \%$, with a median follow-up of 30 months (range, 1-117 months), similar to that in the previous studies. ${ }^{15,23}$

Several studies have reported different prevalence rates for fibrosis/necrosis, teratoma, and viable GCT (Table 4). In a his- 


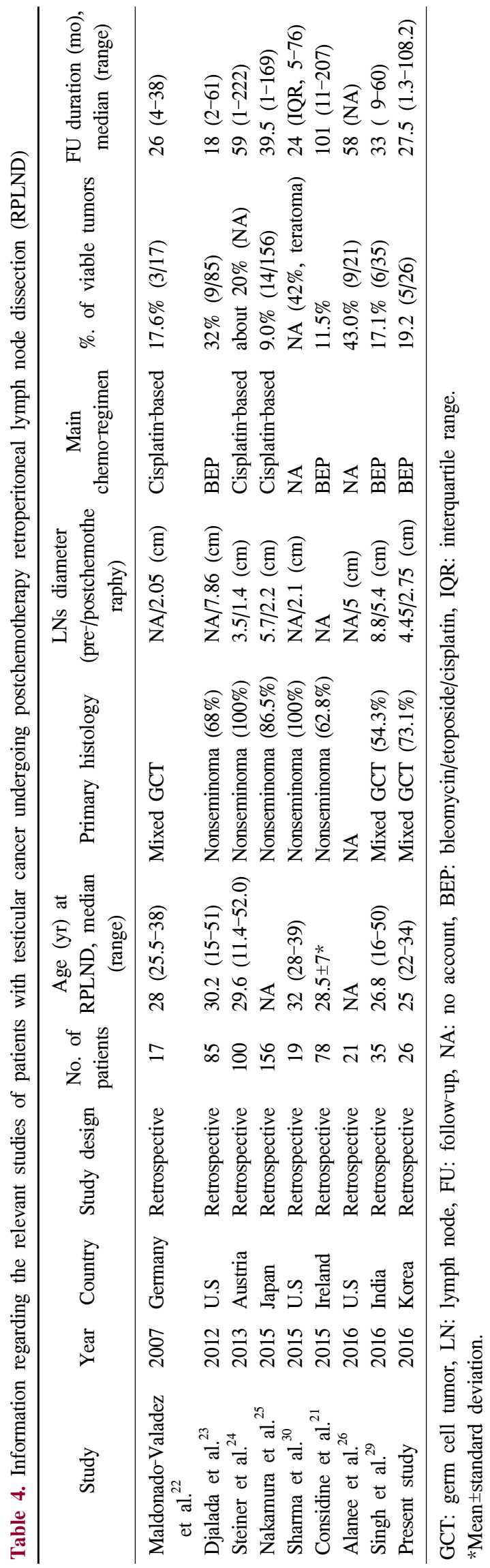

topathological comparison of RPLND specimens after chemotherapy with multiple chemotherapy regimens, Donohue ${ }^{5}$ reported fibrosis in $40 \%-45 \%$ of cases, teratoma in $40 \%-45 \%$ of cases, and viable GCT in $10 \%-20 \%$ of cases. Eggener et al. ${ }^{28}$ and several other groups have also reported fibrosis/necrosis in $35 \%-51 \%$ of cases, teratoma in $21 \%-50 \%$ of cases, and viable GCT in $15 \%-28 \%$ of cases. ${ }^{15}$ In our study, fibrosis/necrosis was present in $42.3 \%$ of cases, teratoma was present in $38.5 \%$ of cases, and viable GCT was present in $19.2 \%$ of cases, similar to the findings in the previous studies. ${ }^{5,15}$ This is important clinical evidence regarding the treatment and prognosis of metastatic testicular carcinoma in Korean men.

Singh et al. ${ }^{29}$ showed that the mean lymph node size before chemotherapy was $8.8 \mathrm{~cm}$ (range, 2.2-20.4) and the size after chemotherapy was reduced to $5.4 \mathrm{~cm}$ (range, $1.2-14.6 \mathrm{~cm}$ ). The sites were the para aortic (40\%), paracaval (34\%), and interaortocaval (23\%) lymph nodes. In our study, the overall lymph node size decreased after chemotherapy. In the case of teratoma, the overall lymph node size did not decrease, but increased instead. The para-aortic and interaortocaval lymph nodes were the most common sites, and paracaval and pelvis were similar (Table 3).

Predictive factors could not be identified in multivariate analysis because of the small sample size. This study is limited by its retrospective design and small sample size. Larger prospective studies in the future are needed to overcome these limitations. However, owing to the low incidence rate of viable GCT, conducting a study with a large sample size remains a challenge. We believe that in spite of these limitations, our study provides valuable insights about testicular cancer.

\section{CONCLUSIONS}

Viable GCT was present in $19.2 \%$ of tumor specimens from patients with testicular cancer who underwent RPLND after chemotherapy, and predictive factors for the presence of viable tumors were not identified. To our knowledge, this is the first study to provide important clinical evidence regarding the treatment and prognosis of metastatic testicular carcinoma in Korean men.

\section{CONFLICT OF INTEREST}

The authors claim no conflicts of interest. 


\section{REFERENCES}

1. Purdue MP, Devesa SS, Sigurdson AJ, McGlynn KA. International patterns and trends in testis cancer incidence. Int J Cancer 2005;115:822-7.

2. Stephenson AJ, Aprikian AG, Gilligan TD, Oldenburg J, Powles T, Toner GC, et al. Management of low-stage nonseminomatous germ cell tumors of testis: SIU/ICUD Consensus Meeting on Germ Cell Tumors (GCT), Shanghai 2009. Urology 2011;78(4 Suppl):S444-55.

3. Filippou P, Ferguson JE 3rd, Nielsen ME. Epidemiology of prostate and testicular cancer. Semin Intervent Radiol 2016; 33:182-5.

4. National Cancer Information Center. Occurrence frequency of testis cancer 2009 [Internet]. Goyang: National Cancer Information Center; 2017 [cited 2016 Oct 23]. Available from: https://www.cancer.go.kr/lay1/program/S1T211C223/cancer/view.do? cancer_seq $=3389 \&$ menu_seq $=3394$.

5. Donohue JP. Evolution of retroperitoneal lymphadenectomy (RPLND) in the management of non-seminomatous testicular cancer (NSGCT). Urol Oncol 2003;21:129-32.

6. Sheinfeld J, Motzer RJ. Stage I testicular cancer management and necessity for surgical expertise. J Clin Oncol 2008;26: 2934-6.

7. Fox EP, Weathers TD, Williams SD, Loehrer PJ, Ulbright TM, Donohue JP, et al. Outcome analysis for patients with persistent nonteratomatous germ cell tumor in postchemotherapy retroperitoneal lymph node dissections. J Clin Oncol 1993;11: 1294-9.

8. Hendry WF, Norman AR, Dearnaley DP, Fisher C, Nicholls J, Huddart RA, et al. Metastatic nonseminomatous germ cell tumors of the testis: results of elective and salvage surgery for patients with residual retroperitoneal masses. Cancer 2002; 94:1668-76.

9. Rick O, Bokemeyer C, Weinknecht S, Schirren J, Pottek T, Hartmann JT, et al. Residual tumor resection after high-dose chemotherapy in patients with relapsed or refractory germ cell cancer. J Clin Oncol 2004;22:3713-9.

10. Stephenson AJ, Tal R, Sheinfeld J. Adjunctive nephrectomy at post-chemotherapy retroperitoneal lymph node dissection for nonseminomatous germ cell testicular cancer. J Urol 2006; 176:1996-9.

11. Stephenson AJ, Bosl GJ, Motzer RJ, Kattan MW, Stasi J, Bajorin DF, et al. Retroperitoneal lymph node dissection for nonseminomatous germ cell testicular cancer: impact of patient selection factors on outcome. J Clin Oncol 2005;23:2781-8.

12. Carver BS, Shayegan B, Serio A, Motzer RJ, Bosl GJ, Sheinfeld J. Long-term clinical outcome after postchemotherapy retroperitoneal lymph node dissection in men with residual teratoma. J Clin Oncol 2007;25:1033-7.
13. Donohue JP, Leviovitch I, Foster RS, Baniel J, Tognoni P. Integration of surgery and systemic therapy: results and principles of integration. Semin Urol Oncol 1998;16:65-71.

14. Comisarow RH, Grabstald H. Re-exploration for retroperitoneal lymph node metastases from testis tumors. J Urol 1976;115:569-71.

15. Eggener SE, Carver BS, Loeb S, Kondagunta GV, Bosl GJ, Sheinfeld J. Pathologic findings and clinical outcome of patients undergoing retroperitoneal lymph node dissection after multiple chemotherapy regimens for metastatic testicular germ cell tumors. Cancer 2007;109:528-35.

16. Albers P, Weissbach L, Krege S, Kliesch S, Hartmann M, Heidenreich A, et al. Prediction of necrosis after chemotherapy of advanced germ cell tumors: results of a prospective multicenter trial of the German Testicular Cancer Study Group. J Urol 2004;171:1835-8.

17. Toner GC, Panicek DM, Heelan RT, Geller NL, Lin SY, Bajorin D, et al. Adjunctive surgery after chemotherapy for nonseminomatous germ cell tumors: recommendations for patient selection. J Clin Oncol 1990;8:1683-94.

18. Gels ME, Nijboer AP, Hoekstra HJ, Sleijfer DT, Molenaar WM, Plukker JT, et al. Complications of the post-chemotherapy resection of retroperitoneal residual tumour mass in patients with non-seminomatous testicular germ cell tumours. Br J Urol 1997;79:263-8.

19. Nowroozi M, Ayati M, Arbab A, Jamshidian H, Ghorbani H, Niroomand $\mathrm{H}$, et al. Postchemotherapy retroperitoneal lymph node dissection in patients with nonseminomatous testicular cancer: a single center experiences. Nephrourol Mon 2015;7:e27343.

20. Baniel J, Foster RS, Rowland RG, Bihrle R, Donohue JP. Complications of post-chemotherapy retroperitoneal lymph node dissection. J Urol 1995;153(3 Pt 2):976-80.

21. Considine S, Heaney R, Conroy R, Thornhill JA. Post-chemotherapy retroperitoneal lymph node dissection in the management of metastatic testis cancer: the 16-year experience in an Irish setting. Ir J Med Sci 2016;185:901-907.

22. Maldonado-Valadez R, Schilling D, Anastasiadis AG, Sturm W, Stenzl A, Corvin S. Post-chemotherapy laparoscopic retroperitoneal lymph-node dissection in testis cancer patients. J Endourol 2007;21:1501-4.

23. Djaladat H, Nichols C, Daneshmand S. Adjuvant surgery in testicular cancer patients undergoing postchemotherapy retroperitoneal lymph node dissection. Ann Surg Oncol 2012;19: 2388-93.

24. Steiner H, Peschel R, Janetschek G, Höltl L, Berger AP, Bartsch G, et al. Long-term results of laparoscopic retroperitoneal lymph node dissection: a single-center 10-year experience. Urology 2004;63:550-5.

25. Nakamura T, Kawauchi A, Oishi M, Ueda T, Shiraishi T, Nakanishi H, et al. Post-chemotherapy laparoscopic retroperitoneal lymph node dissection is feasible for stage IIA/B 
non-seminoma germ cell tumors. Int J Clin Oncol. 2016;21: 791-5.

26. Alanee SR, Carver BS, Feldman DR, Motzer RJ, Bosl GJ, Sheinfeld J. Pelvic lymph node dissection in patients treated for testis cancer: The Memorial Sloan Kettering Cancer Center Experience. Urology 2016;95:128-31.

27. Fléchon A, Tavernier E, Boyle H, Meeus P, Rivoire M, Droz JP. Long-term oncological outcome after post-chemotherapy retroperitoneal lymph node dissection in men with metastatic nonseminomatous germ cell tumour. BJU Int 2010;106:779-85.

28. Eggener SE, Carver BS, Loeb S, Kondagunta GV, Bosl GJ, Sheinfeld J. Pathologic findings and clinical outcome of pa- tients undergoing retroperitoneal lymph node dissection after multiple chemotherapy regimens for metastatic testicular germ cell tumors. Cancer 2007;109:528-35.

29. Singh P, Yadav S, Mahapatra S, Seth A. Outcomes following retroperitoneal lymph node dissection in postchemotherapy residual masses in advanced testicular germ cell tumors. Indian J Urol 2016;32:40-4.

30. Sharma P, Sverrisson EF, Zargar-Shoshtari K, Fishman MN, Sexton WJ, Dickinson SI, et al. Minimally invasive post-chemotherapy retroperitoneal lymph node dissection for nonseminoma. Can J Urol 2015;22:7882-9. 